\title{
Post-operative Pain: An audit of patient reported pain scores after endoscopic and microscopic ear surgery
}

\author{
Rhona McCallum ${ }^{1}$, Mohd Afiq Mohd Slim², and Arunachalam Iyer ${ }^{1}$ \\ ${ }^{1}$ Monklands Hospital \\ ${ }^{2}$ Queen Elizabeth University Hospital Campus
}

August 31, 2021

\begin{abstract}
Objectives The aim of this study was to compare patient reported pain scores and analgesia requirements between endoscopic and microscopic ear surgery. Design Prospective cohort study Setting Secondary care setting from June 2017 to December 2020. Participants Patients undergoing ear surgery performed by a single surgeon. Main outcome measures The primary outcome measure was patient reported pain as recorded by a visual analogue scale (VAS) at days 1,2,3 and 7 post-operatively. Secondary outcome measure was post-operative analgesia requirement. Results Overall, forty-nine patients encompassing 65.3 \% (32/49) endoscopic and 34.7\% (17/49) microscopic procedures were audited. Endoscopic procedures have statistically significant lower VAS pain threshold outcomes when compared against the microscopic procedures on post- operative day one (endoscopic group median VAS 1.5 [0.00;11.5] mm versus microscopic group median VAS 27.0 [15.0;65.0] mm); to day seven (endoscopic group median VAS 0.5 [0.00;2.75] mm versus microscopic group median VAS 9.00 [2.00;52.0] mm). Requirement for analgesia was greater in the microscopic group at day one compared to the endoscopic group $(64.5 \%, 20 / 31$ endoscopic group vs. $100 \%$, $17 / 17$ microscopic; $p=0.004)$. Conclusion Endoscopic ear surgery is less painful and requires less analgesia in the initial postoperative period than microscopic surgery in this cohort. The overall results of this study are useful for pre-operative patient counselling and pain management in the clinical setting. Given the differing applications of microscopic and endoscopic ear surgery, further research is required to study the influence of bone removal, type of operation, and incision type on pain after ear surgery.
\end{abstract}

Post-operative Pain: An audit of patient reported pain scores after endoscopic and microscopic ear surgery

\section{Objectives}

The aim of this study was to compare patient reported pain scores and analgesia requirements between endoscopic and microscopic ear surgery.

\section{Design}

Prospective cohort study

\section{Setting}

Secondary care setting from June 2017 to December 2020.

\section{Participants}

Patients undergoing ear surgery performed by a single surgeon.

\section{Main outcome measures}

The primary outcome measure was patient reported pain as recorded by a visual analogue scale (VAS) at days 1,2,3 and 7 post-operatively. Secondary outcome measure was post-operative analgesia requirement. 


\section{Results}

Overall, forty-nine patients encompassing $65.3 \%$ (32/49) endoscopic and 34.7\% (17/49) microscopic procedures were audited. Endoscopic procedures have statistically significant lower VAS pain threshold outcomes when compared against the microscopic procedures on post- operative day one (endoscopic group median VAS 1.5 [0.00;11.5] mm versus microscopic group median VAS 27.0 [15.0;65.0] mm); to day seven (endoscopic group median VAS $0.5[0.00 ; 2.75] \mathrm{mm}$ versus microscopic group median VAS $9.00[2.00 ; 52.0] \mathrm{mm}$ ). Requirement for analgesia was greater in the microscopic group at day one compared to the endoscopic group (64.5 $\%, 20 / 31$ endoscopic group vs. $100 \%, 17 / 17$ microscopic; $\mathrm{p}=0.004)$.

\section{Conclusion}

Endoscopic ear surgery is less painful and requires less analgesia in the initial post-operative period than microscopic surgery in this cohort. The overall results of this study are useful for pre-operative patient counselling and pain management in the clinical setting. Given the differing applications of microscopic and endoscopic ear surgery, further research is required to study the influence of bone removal, type of operation, and incision type on pain after ear surgery.

\section{5 key points:}

- Endoscopic ear surgery is often described as minimally invasive, an advantage of which may be reduced pain

- We assessed patient reported pain scores between endoscopic and microscopic ear surgery within the first week post-operatively

- Pain scores related to endoscopic ear surgery were lower than those related to microscopic ear surgery in our cohort

- At day one, analgesia requirements were lower in the endoscopic ear surgery group than the microscopic ear surgery group

- Due to the differing applications of endoscopic and microscopic ear surgery, further research is required to study the influence of operation type, bone removal and incision type on pain after ear surgery.

\section{Introduction}

\section{Background}

Endoscopic ear surgery (ESS) has been increasingly adopted into practice over recent years. ${ }^{1}$ The endoscope affords the ability to "look around corners" during middle ear surgery and provides a detailed view of spaces within the middle ear such as the facial recess and anterior epitympanum through the trans-meatal approach. ${ }^{2}$ As such, EES is often described as minimally invasive, with the potential advantages over microscopic surgery of preventing additional bone removal or use of the post-auricular approach in cholesteatoma surgery. ${ }^{3}$ In addition, the endoscope is also being utilised for non-cholesteatoma surgery, with proponents citing that this may reduce the need for ear canal bony removal during myringoplasty, and reduce the potential for chorda tympani injury in stapes surgery. ${ }^{2}$

As with every surgical technique, there is a responsibility to measure and report outcomes. Although it is a widely held belief that EES is less painful that microscopic ear surgery, ${ }^{1}$ there is little evidence to support this supposition. ${ }^{4,5}$ Where pain after endoscopic surgery has been prospectively studied, published literature from Japan and the Czech Republic is in favour of endoscopic surgery as being less painful than microscopic surgery, ${ }^{4,5}$ but recent evidence from the Netherlands has suggested that no link between surgical approach and pain outcomes exist. ${ }^{6}$

\section{OBJECTIVES}

We aim to prospectively compare patient-reported pain scores and analgesia requirements between endoscopic and microscopic ear surgery in our population.

\section{STUDY DESIGN AND SETTING}




\section{Setting}

A prospective audit was undertaken of patient-reported post-operative pain scores after ear surgery performed in the secondary care setting from June 2017 to December 2020. STROBE reporting guidance has been followed.

\section{Operative Details}

Surgery was either total EES, which we classified as our 'endoscopic' group, whereby the endoscope was used as the sole instrument for visualisation, or 'microscopic', whereby the microscope was used for operating with either an endaural or post aural incision. Where the procedure was endoscopic, a $3 \mathrm{~mm}, 14 \mathrm{~cm}$ rigid telescope (Storz®), Tuttlingen, Germany), and a HD 3CCD chip camera (Strykerß, Kalamazoo, USA). Where EES had to be converted to open surgery, this was classified into the microscopic surgery group.

\section{PARTICIPANTS}

Inclusion criteria were patients who underwent ear surgery by a single surgeon within the secondary-care setting. These patients were included if they consented to participate and returned a completed post-operative pain questionnaire. Exclusion criteria included patients six years-old and under, due to concerns regarding ability to accurately report pain via VAS. ${ }^{7}$

\section{Ethical Considerations}

This study was registered and approved as an audit.

\section{Data 'Sources and Measurement}

Patients were asked to record pain on a visual analogue scale (VAS) at days 1, 2,3 and 7 post-operatively. Outcomes recorded were pain on a visual analogue scale, from $0 \mathrm{~mm}$ to $100 \mathrm{~mm}$, where $0 \mathrm{~mm}$ is "no pain" and $100 \mathrm{~mm}$ is "worst pain imaginable". A $100 \mathrm{~mm}$ VAS was selected as this scale is accepted for determining pain outcomes in otolaryngology. ${ }^{8}$ They were given this document to complete after discharge, and then asked to return it at their first follow up outpatient appointment or post it back to the department. In addition, they were asked to fill out a post-operative analgesia diary, with details regarding the date, dose and type of analgesia taken. For purpose of data analysis, analgesia used by patients was categorised into those requiring only simple analgesia or those needing additional opioid analgesia. Simple analgesia encompassed use of non-steroidal anti-inflammatory drugs (NSAIDs) and paracetamol.

Data was recorded prospectively using the Common Otology Audit. ${ }^{9}$ Data used from the database was patient age at time of operation, name of operation, whether procedure was endoscopic or microscopic, International Otology Outcome Group (IOOG) mastoidectomy category where cholesteatoma surgery had taken place, ${ }^{10}$ IOOG middle ear access category where applicable, ${ }^{10}$ and incision type.

\section{MAIN OUTCOME MEASURES}

Primary outcome measure was VAS at days 1,2,3 and 7. Secondary outcome measure was post-operative analgesia requirement.

\section{Subgroup Analysis}

In order to reduce potential sources of bias, for subgroup analysis, patients were divided into those who had reconstructive procedures, which were stapes surgery, myringoplasty and ossiculoplasty; and those who had what we classified as non-reconstructive procedures which included surgery for cholesteatoma and bony meatoplasty. This was similar to the categories used by Salzman et al.$^{5}$ In addition, presence or absence of bone removal was recorded.

\section{Statistical Analysis}

The R language was used to analyse the data. ${ }^{11,12}$ Numerical data was analysed with the Shapiro-Wilk test for normality distribution. The unpaired Two Sample T-test (w), reported as mean \pm standard deviation, 
or Mann-Whitney U-test (u), reported as median [Interquartile Range (IQR)], was used depending on distribution. Categorical data were analysed with Chi-squared test $\left(\mathrm{x}^{2}\right)$ or Fisher's Exact Test (f). Odds ratio (OR) was calculated to determine the degree of association between the type of procedure and analgesia requirement. Sub-analysis based on the aim of the procedure were also performed and analysed with the Kruskal Wallis test. Significance value was set at p [?] 0.05 .

\section{Results}

\section{Patient Demographics}

Overall, forty-nine patients encompassing $65.3 \%$ (32/49) endoscopic and 34.7\% (17/49) microscopic procedures from a single surgeon experience were audited (Table 1). Of these, $61.2 \%$ (30/49) were categorised into the reconstructive group and $38.8 \%$ (19/49) into the non-reconstructive group (Table 1). Most endoscopic procedures were performed for reconstructive purposes $(84.4 \% ; 27 / 32, \mathrm{p}<0.001)$, and the majority of procedures in the microscopic group were performed for non-reconstructive purposes $(82.4 \% ; 14 / 17, \mathrm{p}<0.001$; Table 1). Of the microscopic group, the post-auricular incision was most used $(70.6 \%, 12 / 17)$ followed by the endaural $(23.5,4 / 17)$ incision for access. (Table 1). Of the endoscopic group, all patients had their procedure conducted via the trans-canal route.

\section{$V A S$}

Overall, endoscopic procedures have statistically significant lower VAS pain threshold outcomes when compared against the microscopic procedures on post- operative day one, two, three and seven (Table 2). On subgroup analysis, pain scores were lower for the endoscopic group at day one where procedures were carried out for reconstructive reasons (median VAS $1[0 ; 9] \mathrm{mm}, \mathrm{n}=27$ ) compared to the microscopic group (median VAS $80[45 ; 86.5] \mathrm{mm}, \mathrm{n}=3$ ). This was also the case at day two (endoscopic median VAS $1[0 ; 6.5] \mathrm{mm}, \mathrm{n}=27$, vs. microscopic median VAS 48 [34;55.5] mm, $\mathrm{n}=3$ ) (Figure 1, Supplementary Table 1). This difference did not reach significance at days three and seven (Figure 1, Supplementary Table 1). For the non-reconstructive procedures at all time points, this difference did not reach significance, although there was a trend towards higher pain scores for the microscopic procedures (Figure 1, Supplementary Table 1).

\section{Analgesia Scores}

One patient did not complete their analgesia diary and was therefore excluded. Of the remaining 48 patients, requirement for analgesia was greater in the microscopic group at day one compared to the endoscopic group $(100 \%, 17 / 17$ microscopic group vs. $64.5 \%, 20 / 31$ endoscopic group $\mathrm{p}=0.004)$ (Table 3). Difference in analgesia requirements did not reach significance at each of the other post-operative days studied (Table $3)$. In addition, requirement for opioid analgesia was higher in the microscopic group at day one $(\mathrm{p}=0.001)$ and day two (OR: 6.71; $\mathrm{p}=0.030$ ) although significance diminishes thereafter (Table 4). Post-hoc power calculation based on the findings of a recent study confirmed our study size to have sufficient power $(92.7 \%$ with alpha 0.05). ${ }^{5}$ The minimum sample size requirement is 9 for each arm to reach alpha of 0.05 and power of $80 \%$ to assess the analgesia requirement on post-operative day-1.

\section{Discussion}

\section{Summary of results/Comparison with Literature}

Overall, EES was associated with lower post-operative pain scores than microscopic ear surgery in our population. Our overall results agree with the findings of a group from Japan, who found that pain reported for total EES was significantly lower than for microscopic ear surgery from days 1 to 7 post-operatively. ${ }^{4}$ A study from the Czech Republic of the first four post-operative days, found less pain on VAS at the first post-operative day in their endoscopic group compared to their microscopic group. ${ }^{5}$

Our findings are contradictory to the results of Baazil et al. , who found no difference in pain thresholds between endoscopic and microscopic groups, where pain was measured using the Brief Pain InventoryShort Form (BPI-SF) on a scale of $0-10 .{ }^{6}$ They compared pain after 20 operations carried out by endoscopic transmeatal, microscopic endaural, and retro-auricular approaches, respectively, and found no difference 
between groups. As pain scores were generally low, they concluded that any difference between pain scores were so small as to be of limited clinical relevance. In our study, VAS was reported at a median of 1.5 [0;11.5] mm at post-op day one for the endoscopic group and a median of $27[15 ; 65] \mathrm{mm}$ for the microscopic group. Difference in median results exceeds what is defined as the minimally clinically important difference (MCID) which has been measured for post-operative VAS scores as being $10 \mathrm{~mm} .{ }^{13}$ We therefore believe differences between our groups to be of significance. Of note, operations studied by Baazil et al., within the endoscopic group included tympanoplasty and atticotomy; and those within the microscopic groups included stapedotomy, tympanoplasty, atticotomy, and canal wall up mastoidectomies. ${ }^{6}$ Our study included stapes procedures only within the endoscopic group, and canal wall down mastoidectomy within the microscopic group (Table 1), perhaps explaining our relatively higher pain scores in the microscopic group.

\section{Subgroup analysis}

Salzman et al ., set to address the differing uses of endoscopic and microscopic surgery by dividing operations into reconstructive and cholesteatoma procedures. ${ }^{5}$ All mastoid surgery was excluded. On subgroup analysis, endoscopic cholesteatoma patients suffered from less pain on post-operative day 1 than the microscopic group. Where the procedure was reconstructive, there was no significant difference between groups, but a trend towards less pain within the endoscopic group than the microscopic group. Unlike this study, we did not detect a statistically significant difference in pain scores between our microscopic and endoscopic groups for non-reconstructive procedures, which included both meatoplasty and cholesteatoma surgery; but found a statistically significant reduction in VAS after EES compared to microscopic ear surgery in our reconstructive group at post-operative days 1 and 2. Numbers within our subgroup analysis were small (Supplementary Table 1), therefore these results should be approached with caution. With regards to reconstructive procedures, case series and metanalysis provide some evidence to support that these may be less painful than microscopic surgery whilst being conducted endoscopically. ${ }^{14,15,16}$

\section{Bone removal}

There are indications which exist for microscopic surgery which preclude total EES; for example, if cholesteatoma is present within the mastoid cavity proper, then microscopic ear surgery is recommended for removal of disease. ${ }^{2}$ All mastoid procedures were carried out within our microscopic group (Table 1). This raises the question of whether differing degrees of bone removal account for the difference in pain scores between endoscopic and microscopic groups. Similarly, Kakehata et al. , also reported mastoid surgery only within the microscopic group. ${ }^{4}$ To overcome this, they conducted subgroup analysis between procedures with no bony removal and those with varying degrees of removal. Between these subgroups, no significant difference was detected in pain scores, and it was concluded that bony removal has no influence over pain scores. We also carried out a subgroup analysis based on bone removal and no statistically significant result was found between pain scores between groups. As this subgroup analysis was underpowered $(n=1)$ in the microscopic non-bone removal group), this was not included within our main results (Supplementary Table 2, Supplementary Figure 1). It is therefore difficult to make any statement about the influence of bone removal on our results.

\section{Incision Type}

Another difference between endoscopic and microscopic approaches is the incision type. All but one patient within our microscopic group had endaural or post-aural incision, and this may account for the difference between pain scores. In a previous study, patients undergoing EES reported significantly less scar discomfort, compared to the microscopic ear surgery group, although this group found no difference in overall post-operative pain scores. ${ }^{17}$ Patients were asked to score pain retrospectively at follow-up 2 weeks postoperatively. ${ }^{17}$ The influence of incision type on pain is an area which requires further study.

\section{Analgesia requirements}

We found increased requirement for analgesia in the microscopic group at day 1 post-operatively, and an increased requirement for opioid analgesia at day 1 and day 2 post-operatively in the microscopic group 
(Table 3; Table 4). Kakehata et al. kept patients as inpatients for the course of their stay, and only offered hospital prescribed NSAIDs. ${ }^{4}$ They detected significantly lower analgesia use post-operatively in their endoscopic compared to microscopic group from day 1 to day 7 . Although Salman et al. also found a trend towards lower painkiller use in patients who were operated endoscopically, these results did not reach significance. ${ }^{5}$ Baazil et al. reported that the endaural microscopic group required half as much medication as their retro-auricular microscopic and endaural transmeatal groups, a result which is unexplained. ${ }^{6}$ However, their endoscopic group had a lower requirement for opioids for pain control post-operatively compared to the other groups, consistent with our findings. We therefore believe that the current evidence points towards lesser analgesia requirements after EES.

\section{Limitations}

As stated, a limitation of this study is sample size, which diminishes on subgroup analysis and would be better served by greater patient numbers. As discussed, types of operations in each group differed due to both patient and surgeon factors. Although subgroup analysis has helped to eliminate some of this bias, a randomised control trial would be the best way to measure pain outcomes between groups. Secondly, the number of patients audited was dependent upon patients remembering to fill in and return their forms, which may have opened our audit to bias.

Additionally, a previous study has reported that the threshold below which patients consider themselves recovered postoperatively from surgery to be $33 \mathrm{~mm}$ on VAS. ${ }^{13}$ Overall median VAS scores are lower that this value across our study, with the exception of a median post-operative VAS at day 2 of 36 [20;63] mm for the microscopic group (Table 1). However, as discussed above, the difference between endoscopic and microscopic VAS scores has clinical relevance. Ear surgery has been found to be 4 to 10 ten times less painful in general compared to pain in other subsites of the head and neck, such as oral cavity and pharynx, and therefore our lower VAS scores were not unexpected. ${ }^{8}$ We believe that the generally low VAS scores across our study should not preclude future groups from studying differences in pain between otologic interventions in their population.

\section{Conclusions}

We have found that EES overall is less painful and requires less analgesia in the initial post-operative period than microscopic surgery. Where differing applications of endoscopic and microscopic procedures are accounted for within subgroup analysis, the difference only holds true for reconstructive procedures. It must be acknowledged that the strength of conclusion which can be drawn from subgroup analysis is weak due to small patient numbers. However, the overall results of this study are useful for pre-operative patient counselling and pain management in the clinical setting. Further research is required to study the influence of bone removal, type of operation, and incision type on pain after ear surgery.

\section{Bibliography}

1. Yong M, Mijovic T, Lea J. Endoscopic ear surgery in Canada: a cross-sectional study. Journal of Otolaryngology - Head \& Neck Surgery. 2016 Jan 19;45(1):4.

2. Presutti L, Marchioni D. Endoscopic ear surgery: principles, indications, and techniques. Stuttgart: Thieme; 2015. $403 \mathrm{p}$.

3. Presutti L, Gioacchini FM, Alicandri-Ciufelli M, Villari D, Marchioni D. Results of endoscopic middle ear surgery for cholesteatoma treatment: a systematic review. Acta Otorhinolaryngol Ital. 2014 Jun;34(3):153-7.

4. Kakehata S, Furukawa T, Ito T, Kubota T, Futai K, Watanabe T. Comparison of Postoperative Pain in Patients Following Transcanal Endoscopic Versus Microscopic Ear Surgery. Otol Neurotol. 2018 Aug;39(7):847-53.

5. Salzman R, Bakaj T, Starek I. Postoperative pain and analgesic consumption after endoscopic and microscopic ear procedures. Biomed Pap Med Fac Univ Palacky Olomouc Czech Repub [Internet]. 2021 Feb 4 [cited 2021 Mar 6]; Available from:http://biomed.papers.upol.cz/doi/10.5507/bp.2021.006.html 
6. Baazil AHA, Spronsen E, Ebbens FA, Dikkers FG, De Wolf MJF. Pain After Ear Surgery: A Prospective Evaluation of Endoscopic and Microscopic Approaches. The Laryngoscope. 2020 Sep 18;lary.29038.

7. Shields BJ, Palermo TM, Powers JD, Grewe SD, Smith GA. Predictors of a child's ability to use a visual analogue scale. Child Care Health Dev. 2003 Jul;29(4):281-90.

8. Sommer M, Geurts JWJM, Stessel B, Kessels AGH, Peters ML, Patijn J, et al. Prevalence and Predictors of Postoperative Pain After Ear, Nose, and Throat Surgery. Arch Otolaryngol Head Neck Surg. 2009 Feb 16;135(2):124.

9. Common Otology Audit. Available at : https://www.ear-audit.net. Accessed January $20^{\text {th }} 2021$.

10. Yung M, James A, Merkus P, Philips J, Black B, Tono T, et al. International Otology Outcome Group and the International Consensus on the Categorization of Tympanomastoid Surgery. J Int Adv Otol. 2018 Aug;14(2):216-26.

11. R Core Team. R: A Language and Environment for Statistical Computing [Internet]. 2020. Available from: https://www.R-project.org/

12. Subirana I, Sanz H, Vila J. Building Bivariate Tables: The $\{$ compareGroups $\}$ Package for $\{R\} .2014$. p. 1-16.

13. Myles PS, Myles DB, Galagher W, Boyd D, Chew C, MacDonald N, et al. Measuring acute postoperative pain using the visual analog scale: the minimal clinically important difference and patient acceptable symptom state. British Journal of Anaesthesia. 2017 Mar;118(3):424-9.

14. Surmelioglu O, Ozdemir S, Tarkan O, Tuncer U, Dagkiran M, Cetik F. Endoscopic versus microscopic stapes surgery. Auris Nasus Larynx. 2017 Jun;44(3):253-7

15. Iannella G, Magliulo G. Endoscopic Versus Microscopic Approach in Stapes Surgery: Are Operative Times and Learning Curve Important for Making the Choice? Otology \& Neurotology. 2016 Oct;37(9):1350-7.

16. Manna S, Kaul VF, Gray ML, Wanna GB. Endoscopic Versus Microscopic Middle Ear Surgery: A Meta-analysis of Outcomes Following Tympanoplasty and Stapes Surgery. Otology \& Neurotology. 2019 Sep;40(8):983-93.

17. Baruah P, Bowyer D. Patient-reported outcome measures in forty-three patients undergoing endoscopic middle ear surgery. Clin Otolaryngol. 2019 May;44(3):372-4.

Table 1:

\begin{tabular}{lllll}
\hline & All & Endoscopic & Microscopic & p-value \\
\hline & $\mathrm{n}=49$ & $\mathrm{n}=32$ & $\mathrm{n}=17$ & \\
Age (years-old) & $40.3(16.1)$ & $42.5(15.6)$ & $36.1(16.7)$ & 0.200 \\
Age Category: & & & & 1.000 \\
Adult & $44(89.8 \%)$ & $29(90.6 \%)$ & $15(88.2 \%)$ & \\
Child & $5(10.2 \%)$ & $3(9.38 \%)$ & $2(11.8 \%)$ & 0.917 \\
Gender: & & & & \\
Female & $24(49.0 \%)$ & $15(46.9 \%)$ & $9(52.9 \%)$ & \\
Male & $25(51.0 \%)$ & $17(53.1 \%)$ & $8(47.1 \%)$ & $<0.001$ \\
Bone Removal: & & & & \\
No & $27(55.1 \%)$ & $26(81.2 \%)$ & $1(5.9 \%)$ & \\
Yes & $22(44.9 \%)$ & $6(18.8 \%)$ & $16(94.1 \%)$ & \\
Procedure aim: & & & & \\
Non-Reconstructive Procedure & $19(38.8 \%)$ & $5(15.6 \%)$ & $14(82.4 \%)$ & \\
Reconstructive Procedure & $30(61.2 \%)$ & $27(84.4 \%)$ & $3(17.6 \%)$ \\
Name of Operation: & & & & \\
Attico-antrostomy & $2(4.1 \%)$ & $1(3.12 \%)$ & $1(5.9 \%)$ \\
Atticotomy & $5(10.2 \%)$ & $4(12.5 \%)$ & $1(5.9 \%)$ & \\
Bony Meatoplasty & $4(8.2 \%)$ & $0(0 \%)$ & $4(23.5 \%)$ & \\
Mastoidectomy with canal wall preserved & $1(2.0 \%)$ & $0(0 \%)$ & $1(5.9 \%)$ \\
Mastoidectomy with canal wall preserved plus atticotomy & $1(2.0 \%)$ & $0(0 \%)$ & $1(5.9 \%)$
\end{tabular}




\begin{tabular}{|c|c|c|c|c|}
\hline & All & Endoscopic & Microscopic & p-value \\
\hline Mastoidectomy with canal wall preserved plus posterior tymp & $3(6.1 \%)$ & $0(0 \%)$ & $3(17.6 \%)$ & \\
\hline Modified radical mastoidectomy & $3(6.1 \%)$ & $0(0 \%)$ & $3(17.6 \%)$ & \\
\hline Myringoplasty & $9(18.4 \%)$ & $8(25.0 \%)$ & $1(5.9 \%)$ & \\
\hline Ossiculoplasty & $6(12.2 \%)$ & $4(12.5 \%)$ & $2(11.8 \%)$ & \\
\hline Stapedectomy & $15(30.6 \%)$ & $15(46.9 \%)$ & $0(0 \%)$ & \\
\hline \multicolumn{5}{|l|}{ Incision Type: } \\
\hline Endaural & $4(8.16 \%)$ & $0(0 \%)$ & $4(23.5 \%)$ & \\
\hline Post-aural & $12(24.5 \%)$ & $0(0 \%)$ & $12(70.6 \%)$ & \\
\hline No incision & $3(6.1 \%)$ & $32(100 \%)$ & $1(5.9 \%)$ & \\
\hline
\end{tabular}

Table 2:

\begin{tabular}{lllll}
\hline & All & Endoscopic & Microscopic & p-overall \\
\hline & $\mathrm{n}=49$ & $\mathrm{n}=32$ & $\mathrm{n}=17$ & \\
VAS Post-op Day-1 $(\mathrm{mm})$ & $8[0.00 ; 22.0]$ & $1.50[0.00 ; 11.5]$ & $27.0[15.0 ; 65.0]$ & $<0.001$ \\
VAS Post-op Day-2 $(\mathrm{mm})$ & $5.00[0.00 ; 35.0]$ & $1.50[0.00 ; 9.25]$ & $36.0[20.0 ; 63.0]$ & $<0.001$ \\
VAS Post-op Day-3 $(\mathrm{mm})$ & $2.00[0.00 ; 22.0]$ & $0.00[0.00 ; 9.50]$ & $24.0[12.0 ; 71.0]$ & $<0.001$ \\
VAS Post-op Day-7 $(\mathrm{mm})$ & $2.00[0.00 ; 9.50]$ & $0.50[0.00 ; 2.75]$ & $9.00[2.00 ; 52.0]$ & 0.003 \\
\hline
\end{tabular}

Table 3

\begin{tabular}{|c|c|c|c|c|c|c|}
\hline & All & Endoscopic & Microscopic & OR & $\mathrm{p}$ ratio & p overall \\
\hline & $\mathrm{n}=48$ & $\mathrm{n}=31$ & $\mathrm{n}=17$ & & & \\
\hline Day 1 analgesia requirement: & & & & & & 0.004 \\
\hline None & $11(22.9 \%)$ & $11(35.5 \%)$ & $0(0.00 \%)$ & & & \\
\hline Yes & $37(77.1 \%)$ & $20(64.5 \%)$ & $17(100 \%)$ & & & \\
\hline Day 2 analgesia requirement: & & & & & & 0.073 \\
\hline None & $18(37.5 \%)$ & $15(48.4 \%)$ & $3(17.6 \%)$ & & & \\
\hline Yes & $30(62.5 \%)$ & $16(51.6 \%)$ & $14(82.4 \%)$ & $4.12[1.06 ; 21.7]$ & 0.040 & \\
\hline Day 3 analgesia requirement: & & & & & & 0.227 \\
\hline None & $24(50.0 \%)$ & $18(58.1 \%)$ & $6(35.3 \%)$ & & & \\
\hline Yes & $24(50.0 \%)$ & $13(41.9 \%)$ & $11(64.7 \%)$ & $2.47[0.73 ; 9.04]$ & 0.148 & \\
\hline Day 7 analgesia requirement: & & & & & & 0.080 \\
\hline None & $41(85.4 \%)$ & $29(93.5 \%)$ & $12(70.6 \%)$ & & & \\
\hline Yes & $7(14.6 \%)$ & $2(6.45 \%)$ & $5(29.4 \%)$ & $5.58[1.00 ; 48.2]$ & 0.050 & \\
\hline
\end{tabular}

Table 4:

\begin{tabular}{|c|c|c|c|c|c|c|}
\hline & All & Endoscopic & Microscopic & OR & $p$ ratio & p overall \\
\hline & $\mathrm{n}=48$ & $\mathrm{n}=31$ & $\mathrm{n}=17$ & & & \\
\hline Day 1: & & & & & & 0.001 \\
\hline None & $11(22.9 \%)$ & $11(35.5 \%)$ & $0(0.00 \%)$ & & & \\
\hline Simple Analgesia & $23(47.9 \%)$ & $16(51.6 \%)$ & $7(41.2 \%)$ & & & \\
\hline Opioid Analgesia & $14(29.2 \%)$ & $4(12.9 \%)$ & $10(58.8 \%)$ & & & \\
\hline Day 2: & & & & & & 0.060 \\
\hline None & $18(37.5 \%)$ & $15(48.4 \%)$ & $3(17.6 \%)$ & & & \\
\hline
\end{tabular}




\begin{tabular}{lllllll}
\hline & All & Endoscopic & Microscopic & OR & p ratio & p overall \\
\hline Simple Analgesia & $20(41.7 \%)$ & $12(38.7 \%)$ & $8(47.1 \%)$ & $3.15[0.71 ; 18.0]$ & 0.134 & \\
Opioid Analgesia & $10(20.8 \%)$ & $4(12.9 \%)$ & $6(35.3 \%)$ & $6.71[1.19 ; 48.4]$ & 0.030 & \\
Day 3: & & & & & 0.151 \\
None & $24(50.0 \%)$ & $18(58.1 \%)$ & $6(35.3 \%)$ & & & \\
Simple Analgesia & $16(33.3 \%)$ & $10(32.3 \%)$ & $6(35.3 \%)$ & $1.77[0.43 ; 7.38]$ & 0.425 & \\
Opioid Analgesia & $8(16.7 \%)$ & $3(9.68 \%)$ & $5(29.4 \%)$ & $4.64[0.84 ; 30.7]$ & 0.078 & \\
Day 7: & & & & & 0.074 \\
None & $41(85.4 \%)$ & $29(93.5 \%)$ & $12(70.6 \%)$ & & & \\
Simple Analgesia & $4(8.33 \%)$ & $1(3.23 \%)$ & $3(17.6 \%)$ & $6.40[0.67 ; 197]$ & 0.110 & \\
Opioid Analgesia & $3(6.25 \%)$ & $1(3.23 \%)$ & $2(11.8 \%)$ & $4.39[0.33 ; 147]$ & 0.261 & \\
\hline
\end{tabular}

Figure and Table Legends:

Table 1: Baseline demographics and procedural details for endoscopic and microscopic ear surgery groups

Table 2: Median Visual Analogue Scale (VAS) results at post-operative days 1,2,3 and 7, where $0 \mathrm{~mm}$ represents "no pain" and $100 \mathrm{~mm}$ represents "worst pain imaginable" . Interquartile range is represented in brackets.

Figure 1: Subgroup analysis. Postoperative pain score with visual analogue scores at post-operative day 1, 2, 3 and 7. Procedures are split into those with reconstructive aim (stapes surgery, ossiculoplasty, myringoplasty) versus those in non-reconstructive group (cholesteatoma surgery and meatoplasty); for both endoscopic and open ear surgery groups

Table 3: Analgesia requirement at day 1,2,3 and 7 post-operatively for endoscopic and microscopic groups. $\mathrm{OR}=$ odds ratio

Table 4: Analgesia requirement at day 1,2,3 and 7 post-operatively for endoscopic and microscopic groups. Simple analgesia encompasses non-steroidal anti-inflammatory drugs (NSAIDs) and paracetamol. OR= odds ratio

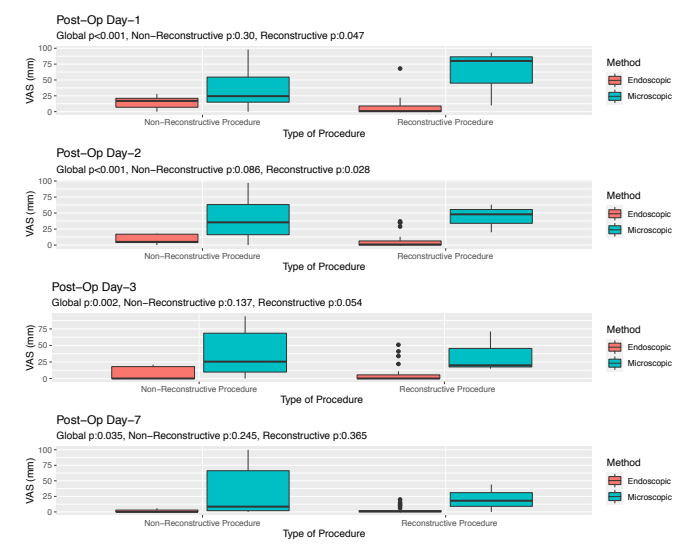

